\author{
Leonidas Grigorakos $^{1,2 *}$, Ioannis \\ Nikolopoulos ${ }^{3}$, Katerina \\ Tzortzopoulou², Anastasia \\ Alexopoulou $^{2}$, Mariza Gioka ${ }^{2}$ and \\ Dimitrios Veldekis ${ }^{3}$
}

${ }^{1}$ Associate Professor of Medicine (ICU), Faculty of Nursing, National and Kapodistrian University of

Athens

${ }^{2}$ Intensive Care at Trauma, Hospital of Athens, KAT,

Kifissia, Athens, Greece

${ }^{3}$ Sotiria Regional Chest Diseases Hospital of Athens

Dates: Received: 28 May, 2015; Accepted: 05

June, 2015; Published: 09 June, 2015

*Corresponding author: Leonidas Grigorakos $\mathrm{MD}, \mathrm{PhD}$, Associate Professor of Medicine, $36 \mathrm{~B}$ Figaleias Str, 14564, N. Kifissia, Athens, Greece. Tel: +30.210.3709522; Fax: +30.210.3709520; E-mail: grigorakos@parliament.gr

www.peertechz.com

Keywords: Asthma attacks; Pregnancy; Medication; $\mathrm{ICU}$

\author{
Case Report
}

\section{Asthma Attacks During Pregnancy in the ICU: Just Be Alert}

\section{Abstract}

Background: Asthma represents one of the most common medical conditions encountered during pregnancy, which may seriously affect its outcome.

Objective: To evaluate the outcome of asthma attacks during pregnancy and the role medications play for appropriate asthma management.

Methods: In this prospective study, we present our 15 years' experience from hospitalization in ICU of 11 pregnant women (13-29 weeks of gestation) with severe asthma.

Results: All patients had a favorable outcome after being applied proper medical procedures and medications. The mean duration of patients' stay in ICU stay was 4.6 days, they were all closely observed until birth, and none of them had further asthma attacks during pregnancy after discharge.

Conclusions: In our study severe asthma in pregnant women had an unexpectedly favorable outcome. Therefore, proper medication seems to be of crucial importance for positive outcomes in the case of asthma attacks during pregnancy. Moreover, careful follow-up by clinicians experienced in managing asthma seems to be essential for pregnant asthmatic patients.
\end{abstract}

\section{Background}

Asthma represents one of the most common medical conditions encountered during pregnancy, which occurs in 3 to $8 \%$ of pregnant women [1]. Pregnancy may be associated with changes in the course of asthma, and asthma may affect the outcome of pregnancy. Although the incidence of severe asthma during pregnancy is low (0.4\%-1.3\%), its consequences on mother and fetus can be disastrous [2]. Albeit small, significant increase in complications of pregnancy in asthmatic women such as perinatal mortality, pre-eclampsia, preterm deliveries or low birth weight infants are being registered [3]. Moreover, patients with more severe asthma have a 30 to 100 percent increased risk of severe exacerbation [4]. While the recommended pharmacotherapy of acute asthma during pregnancy does not differ substantially from the management in non-pregnant patients, intensive monitoring of both mother and fetus is essential $[5,6]$.

\section{Methods}

The prospective study included 11 nonsmoking pregnant women (13-29 weeks of gestation) with severe asthma admitted to our ICU between January 2000 and July 2014 through the emergency department of our hospital. Severe asthma was defined as an exacerbation of asthma which was not controlled by the patient's normal rescue medication and which was treated as an emergency [7]. All patients were admitted to ICU because they reached standard criteria of admission [8]. All 11 women were admitted in ICU on the basis of pre-admission performed skinprick tests (SPT) where they were classified as atopic ( 8 women - 72\%) and non-atopic (3 women - 28\%) [9]. Patients' ages range from 19 to 40 years. The study was approved by the institutional board throughout all treatment, and informed consent was obtained from all patients.

\section{Results}

All patients had a previous history of asthma and had experienced a severe acute asthma attack without response to the usual outpatient treatment before being admitted to the ICU. Admission to our ICU occurred within a period of 3 hours after failure of usual outpatient treatment and deterioration of symptoms.

On physical examination all patients (100\%) had dyspnea, were diaphoretic and had inspiratory and expiratory wheezes on both chest sided. Seven (63.6\%) patients had sinus tachycardia without other ECG's abnormalities (>100 beats/min). 11 (100\%) patients had tachypnea (>30 breaths/min), $3(27.2 \%)$ patients had a temperature of $38^{\circ} \mathrm{C}, 9(81.8 \%)$ patients had elevated WBC $(>12.800 / \mathrm{mm} 3)$ while abnormal arterial blood gases $(\mathrm{PaO} 2<65 \mathrm{~mm} \mathrm{Hg}, \mathrm{PaCO} 2<35 \mathrm{~mm}$ $\mathrm{Hg}, \mathrm{PH}>7.48$ on room air) were detected in all the cases (100\%) (Table 1). No Chest X-RAY were performed because of pregnancy status. Shortly after admission, all patients demonstrated at spirometry a severe obstructive ventilator defect that was partially relieved by bronchodilators and use of inhaled steroids. All patients were treated

\begin{tabular}{|l|l|}
\hline Table 1: Signs, Symptoms and Laboratory tests. \\
\hline Dyspnea & 11 patients $(100 \%)$ \\
\hline Diaphoresis & 11 patients $(100 \%)$ \\
\hline Wheezes & 11 patients $(100 \%)$ \\
\hline Tachycardia & 7 patients $(63.6 \%)$ \\
\hline Tachypnea & 11 patients $(100 \%)$ \\
\hline Fever & 3 patients $(27.2 \%)$ \\
\hline Elevated WBC & 9 patients $(81.8 \%)$ \\
\hline Abnormal ABGs & 11 patients $(100 \%)$ \\
\hline
\end{tabular}


successfully using a standard protocol with subcutaneous epinephrine $0.3-0.5 \mathrm{ml}$ of a $1: 1000$ solution given every 15 to 20 minutes, IV aminophylline at recommended doses, hydrocortisone $250 \mathrm{mg}$ every six hours, oxygen by venturi mask, nebulized salbutamol, ipratropium and steroids as needed. Clinicians experienced in managing asthma in contact with obstetricians, psychiatrists, and trained nurses treated our patients. No adverse effects or complications were noted during treatment and until birth, neither for patients nor for fetus.

The mean duration of ICU stay was 4.6 days while the mean hospitalization time was 11.2 days. Upon discharge, all patients were prescribed preventive medications (i.e. long acting salmeterol with flexotide) and had no further asthma attacks.

\section{Discussion}

Objective monitoring of maternal lung function and fetal wellbeing as a guide to therapy, proper control of environmental and other triggers of asthma, patient education as well as pharmacologic therapy are stressed to be the most important components of effective therapy in situations of asthma during pregnancy [10]. Together, careful follow-up by clinicians with long experience in managing asthma as well as effective communication among clinician managing the asthma, patient and the obstetrician seems to be essential for a positive outcome [11]. More so, even though data regarding the use of oral glucocorticoids during pregnancy have not been usually reassuring, benefit-risk considerations still favor their use in patients with severe asthma exacerbations [12]. More precise, most medications used in the treatment of asthma fall into categories B or C [13]. While serious concern has been raised about the possibility that the alpha adrenergic effect of epinephrine might cause vasoconstriction in the uteroplacental circulation, the safety data on inhaled glucocorticoids are reassuring [14]. Moreover, extensive clinical experience suggests that theophylline and its ethylenediamine complex, aminophylline, are safe during pregnancy $[15,16]$. Even though the recommended pharmacotherapy of acute asthma during pregnancy does not differ substantially from the management in non-pregnant patients [17], intensive monitoring of both mother and fetus is essential. The changes in blood gases which occur secondary to acute asthma during pregnancy will be superimposed on the "normal" respiratory alkalosis of pregnancy. Thus, a pCO $2<35 \mathrm{~mm} \mathrm{Hg}$ and pO $2<65 \mathrm{~mm}$ $\mathrm{Hg}$, associated with acute asthma represent more severe compromise during pregnancy than in the non-gravid state [18].

In conclusion, the unexpectedly favorable outcome of severe asthma attacks in the case of pregnant women hospitalized in our ICU seems to be positively related with proper treatment and medications in specialized centers provided by experienced physicians using standard protocols and until the therapeutic effects are obtained.
Moreover, careful follow-up by clinicians experienced in managing asthma seems to be essential for pregnant asthmatic patients during pregnancy.

\section{References}

1. Namazy JA, Schatz M (2005) Pregnancy and asthma: recent developments. Curr Opin Pulm Med 11: 56-60.

2. Tan KS, Thompson NC (2000) Asthma in pregnancy. Am J Med 109: $727-$ 733.

3. Grewal P, Mulla ZD, Simon MR (2008) Effect of Maternal Asthma on Pregnancy Outcomes: An Analysis of Texas Hospital Data. AEP 18: 735-736.

4. Schatz M (1999) Asthma and pregnancy. Lancet 353: 1202-1204.

5. Holland SM, Thompson KD (2006) Acute severe asthma presenting in late pregnancy. Int $\mathrm{J}$ of Obst Anest 15: 75-78.

6. Vanders RL, Murphy VE (2015) Maternal complications and the management of asthma in pregnancy. Women's Health 11: 183-191.

7. Bel EH (2004) Clinical phenotypes of asthma. Curr Opin Pulm Med 10: 44-50

8. Jain DG, Singal RK, Jain GB, Clark RA, Chauhan SS (2006) Understanding and Managing Acute Severe and Difficult Asthma. JIACM 7: 316-327.

9. Stenius-Aarniala B, Piirila P, Teramo K (1988) Asthma and pregnancy: a prospective study of 198 pregnancies. Thorax 43: 12-18.

10. National Asthma Education Prevention Program (NAEPP) (2004) Working Group Report on Managing Asthma during Pregnancy: Recommendations for Pharmacologic Treatment? Update (NAEPP 2005). NIH Publication No. 05-5236. Rockville MD, U.S. Department of Health and Human Services, National Institutes of Health, National Heart, Lung, and Blood Institute, March.

11. Louik C, Schatz M, Hernández-Díaz S, Werler MM, Mitchell AA (2010) Asthma in pregnancy and its pharmacologic treatment. Ann Allergy Asthma Immunol 105: 110-117.

12. Hasegawa K, Cydulka RK, Sullivan AF, Langdorf MI, Nonas SA, et al. (2015) Improved management of acute asthma among pregnant women presenting to the ED. Chest 147: 406-414.

13. Schatz M (2001) The efficacy and safety of asthma medication during pregnancy. Semin Perinatol 25:145-152.

14. Namazy JA, Schatz M (2004) Update in the treatment of asthma during pregnancy. Clin Rev Allergy Immunol 26: 139-148.

15. Dombrowski MP, Schatz M (2008) ACOG practice bulletin: clinical management guidelines for obstetrician-gynecologists number 90, February 2008: asthma in pregnancy. Obstet Gynecol 111: 457-464.

16. Rocklin RE (2011) Asthma, asthma medications and their effects on maternal/ fetal outcomes during pregnancy. Reproductive Toxicology 32: 189-197.

17. National Heart, Lung, and Blood Institute (2005) NAEPP expert panel report. Managing asthma during pregnancy: recommendations for pharmacologic treatment - 2004 update. J Allergy Clin Immunol 115: 34-46.

18. Petitti DB (2000) Perinatal epidemiology: Studying the effects of illness and medications during pregnancy. Immunol Allergy Clin North Am 20: 673-685. 